\title{
Serotonin transporter genotype 5HTTLPR as a marker of differential susceptibility? A meta-analysis of child and adolescent gene-by-environment studies
}

\author{
MH van IJzendoorn ${ }^{1}, \mathrm{~J}$ Belsky ${ }^{2}$ and MJ Bakermans-Kranenburg ${ }^{1}$
}

\begin{abstract}
We present results of a meta-analysis of gene-by-environment $(G \times E)$ studies involving the serotonin transporter genotype 5HTTLPR to evaluate empirical support for two competing conceptual frameworks in developmental psychopathology: diathesis-stress and differential susceptibility. From a diathesis-stress perspective, the cumulative negative effects of the short allele (ss and sl genotypes) and adverse environments on development have been stressed. From a differential-susceptibility perspective, carriers of the $s$ allele are predicted to be more open to adverse as well as positive environments, for better and for worse. Studies with children and adolescents up to 18 years of age $(N=9361)$ were included. We found 41 effect sizes $(N=5863)$ for the association between negative environments and developmental outcomes with or without significant moderation by 5HTTLPR genotype and 36 effect sizes $(N=3498)$ for the potentially 5HTTLPR-moderated association between positive environments and developmental outcomes. Five moderators were examined: age, ethnicity, genotyping (biallelic or triallelic) and methods used to assess environment and outcome. In the total set of studies, including studies with mixed ethnicities, we found that ss/sl carriers were significantly more vulnerable to negative environments than II carriers, thus supporting the diathesis-stress model. In the Caucasian samples, however, ss/sl carriers also profited significantly more from positive environmental input than II carriers. Associations between (positive or negative) environment and (positive or negative) developmental outcome were absent for II carriers. The meta-analytic findings support the hypothesis that in Caucasian samples 5HTTLPR is a genetic marker of differential susceptibility. $\mathbf{G} \times \mathbf{E}$ interactions might be critically dependent on ethnicity.

Translational Psychiatry (2012) 2, e147; doi:10.1038/tp.2012.73; published online 7 August 2012
\end{abstract}

\section{Introduction}

Most gene-by-environment $(\mathrm{G} \times \mathrm{E})$ studies of the interaction of measured genes by measured environments emphasize the cumulative negative effects of specific 'risk' genes and adverse environments, whereas potentially cumulative positive effects of the same 'risk' genes (better called 'susceptibility' or 'plasticity' genes) interacting with positive environments remain understudied. $^{1-4}$ From a diathesisstress perspective, the potentially cumulative negative effects of the 5HTTLPR short allele (ss and sl genotypes) and adverse environments have been stressed in many studies, as in the association between negative childhood experiences and adult depression. ${ }^{5-7}$ The differential-susceptibility perspective highlights the need to examine the 5HTTLPRmoderated associations between negative and positive environmental influences and developmental outcomes from the position that certain individuals are not just more vulnerable to adversity because of their genetic make-up, but disproportionately responsive to positive and negative environmental experiences and exposures. Here we present the first meta-analytic evidence addressing the question whether the 5HTTLPR genotype should be considered a marker of vulnerability or susceptibility.
Central to the differential-susceptibility hypothesis is the proposition that individuals vary in their susceptibility to the same environmental influences. From an evolutionary perspective, it seems implausible that 'risk' alleles would have survived if they did not promote fitness in some circumstances or contexts. ${ }^{8}$ It is thus a bold but also intuitively plausible conjecture that widespread human characteristics such as a 'reactive' temperament or a 'risky' genotype are, in fact, markers of susceptibility to positive and negative circumstances, for better and for worse..$^{2,9,10}$ Susceptibility markers would not have emerged, survived and spread across a substantial minority of the population if they did not advance adaptation to at least some ecological niches for at least some individuals, ${ }^{10}$ although random genetic drift cannot be excluded as a possible explanation of their existence. Recent years have witnessed a growing body of correlational evidence consistent with differential-susceptibility thinking. ${ }^{3}$ The first such work documenting genetic differential susceptibility focused on the dopamine D4 receptor gene, ${ }^{11}$ with a recent meta-analysis of $\mathrm{G} \times \mathrm{E}$ studies involving dopamine-related genes and children under 10 years of age indicating that associations between positive environments and positive developmental outcomes were as strong as the negative associations in case of the

${ }^{1}$ Centre for Child and Family Studies, Leiden University, Leiden, The Netherlands and ${ }^{2}$ University of California at Davis, King Abdulaziz University, Birkbeck University of London, Jeddah, Saudi Arabia

Correspondence: Dr MH van IJzendoorn or Dr MJ Bakermans-Kranenburg, Centre for Child and Family Studies, Leiden University, PO Box 9555, Leiden 2300 RB, Netherlands.

E-mail: vanijzen@fsw.leidenuniv.nl or E-mail: bakermans@fsw.leidenuniv.nl

Keywords: adolescents; children; diathesis stress; differential susceptibility; 5 HTTLPR; meta-analysis; serotonin

Received 11 April 2012; revised 11 May 2012; accepted 04 July 2012 
so-called 'risk' polymorphisms. ${ }^{12}$ Here we test whether the same is the case for the short versus long allele of 5HTTLPR, focusing on children under the age of 18 .

\section{Hypotheses}

A series of meta-analyses tested two contrasting hypotheses about the role of 5 HTTLPR in $\mathrm{G} \times \mathrm{E}$ interactions. The dominant diathesis-stress or cumulative-risk hypothesis stipulates that carriers of the $s$ allele are more vulnerable to the negative effects of adverse environments (double risk). The emerging differential-susceptibility model predicts that carriers of the s-allele are not only more vulnerable to the influence of negative environments but also profit more from the beneficial influences of positive environments. From a differential-susceptibility perspective, we expect the interactive effects of the 5HTTLPR genotype and positive environments on development to be as large as the interaction effects of the same genotype and risk environments.

\section{Materials and methods}

Data sources. For our meta-analysis, we systematically searched the PsycLit, Medline and ISI web of knowledge databases, with the key words 'serotonin' or '5HTT*', and 'human' in the title or abstract. The asterisk indicates that the search contained the word or word fragment. In addition, references of three recently published review papers on the same topic were scrutinized. ${ }^{3,4,10}$

Table 1 Characteristics of studies included in the meta-analyses

\begin{tabular}{|c|c|c|c|c|c|c|c|}
\hline \multirow[t]{2}{*}{ Study } & \multirow[t]{2}{*}{$\mathbf{N}$} & \multirow{2}{*}{$\begin{array}{l}\text { Positive/ } \\
\text { negative }^{\mathrm{a}}\end{array}$} & \multirow[t]{2}{*}{ Age } & \multirow[t]{2}{*}{ Ethnicity ${ }^{b}$} & \multirow{2}{*}{$\begin{array}{c}\text { Genotyping } \\
\text { triallelic }\end{array}$} & \multicolumn{2}{|c|}{ Assessment } \\
\hline & & & & & & environment & outcome \\
\hline Bakermans et al. (2012) & 37 & Negative & $<10$ & Caucasian & No & Observation & Observation \\
\hline Benjet et al. (2010) & 78 & Negative & $\geqslant 10$ & Mixed & No & Self-report & Self-report \\
\hline Brody et al. (2009) & 419 & Positive & $\geqslant 10$ & Mixed & No & Observation & Self-report \\
\hline Cicchetti et al. (2010) & 850 & Negative & $<10$ & Mixed & No & Observation & Self-report \\
\hline Cicchetti et al. (2011) & 92 & Positive & $<10$ & Mixed & No & Observation & Observation \\
\hline Drury et al. (2012) & 100 & Positive & $<10$ & Caucasian & No & Observation & Other report \\
\hline Eley et al. (2004) & 220 & Negative & $\geqslant 10$ & Caucasian & No & Other report & Self-report \\
\hline Eley et al. (2011) & 344 & Positive & $<10$ & Caucasian & No & Observation & Observation \\
\hline Fox et al. (2005) & 73 & Positive & $<10$ & Caucasian & No & Other report & Observation \\
\hline Gibb et al. (2011) & 74 & Negative & $<10$ & Caucasian & No & Observation & Observation \\
\hline Gilissen et al. (2008) & 87 & Positive & $<10$ & Caucasian & No & Observation & Observation \\
\hline Hankin et al. (2012) & 220 & Negative & $\geqslant 10$ & Mixed & Yes & Other report & Self-report \\
\hline Ivorra et al. (2010) & 317 & Negative & $<10$ & Caucasian & No & Other report & Other report \\
\hline Jacobs et al. (2011) & 123 & Negative & $\geqslant 10$ & Caucasian & Yes & Other report & Observation \\
\hline Kaufman et al. (2004) & 101 & Negative & $\geqslant 10$ & Mixed & No & Observation & Self-report \\
\hline Kochanska et al. (2011) & 88 & Positive & $<10$ & Caucasian & No & Observation & Other report \\
\hline Luijk et al. (2011) & 512 & Positive & $<10$ & Caucasian & No & Observation & Observation \\
\hline Kumsta et al. (2010) & 64 & Negative & $\geqslant 10$ & Caucasian & No & Observation & Other report \\
\hline Mueller et al. (2011) & 115 & Negative & $<10$ & Caucasian & Yes & Self-report & Observation \\
\hline Nijmeijer et al. (2010) & 194 & Negative & $\geqslant 10$ & Caucasian & No & Other report & Other report \\
\hline Nilsson et al. (2005) & 196 & Positive & $\geqslant 10$ & Caucasian & No & Self-report & Self-report \\
\hline Nobile et al. (2007) & 689 & Negative & $\geqslant 10$ & Caucasian & No & Other report & Other report \\
\hline Paaver et al. (2008) & 435 & Positive & $\geqslant 10$ & Caucasian & No & Self-report & Self-report \\
\hline Pauli-Pott et al. (2009) & 69 & Positive & $<10$ & Caucasian & No & Observation & Observation \\
\hline Pluess et al. (2011) & 1513 & Negative & $<10$ & Caucasian & No & Other report & Other report \\
\hline Sadeh et al. (2010) & 296 & Positive & $\geqslant 10$ & Mixed & No & Other report & self-report \\
\hline Sonuga et al. (2009) & 681 & Positive & $\geqslant 10$ & Caucasian & No & Observation & Other report \\
\hline Spangler et al. (2009) & 94 & Negative & $<10$ & Caucasian & No & Observation & Observation \\
\hline Sugden et al. (2010) & 1174 & Negative & $\geqslant 10$ & Caucasian & No & Self-report & Other report \\
\hline Sulik et al. (2012) & 106 & Positive & $<10$ & Caucasian & No & Observation & Other report \\
\hline
\end{tabular}

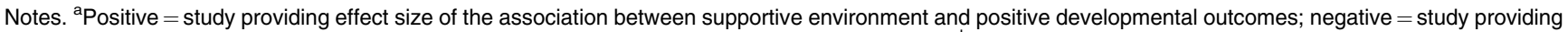
effect size of the association between adverse environment and negative developmental outcome. ${ }^{\mathrm{b}} \mathrm{Caucasian}=>80 \%$ of $\mathrm{the}$ sample Caucasian.
Study selection. The search was restricted to studies with behavioral, psychiatric or developmental outcomes for children under the age of 18 years, thereby excluding purely medical or physical parameters of child development as outcomes for analysis. We finished the search on 1 March 2012. Medical treatment, as an environmental factor, and also nonempirical papers or papers with insufficient statistics were excluded. Only refereed reports in the English language were included. Studies reporting the presence or absence of a significant $\mathrm{G} \times \mathrm{E}$ interaction with $5 \mathrm{HTTLPR}$ were included even if the main goal of the research was the presentation of a genetic or environmental main effect. Therefore the literature search was 'blind' for the kind of $\mathrm{G} \times \mathrm{E}$ outcome and unbiased as to theoretical perspective (see Table 1 for a list of studies).

Data extraction. We identified 77 pertinent effect sizes on 9361 subjects from 30 reports, providing data for two metaanalyses on the moderating role of 5HTTLPR, when it comes to the impact of the environment on development. Forty-one effect sizes $(N=5863)$ concerned vulnerability, that is, moderation by 'risk alleles' (ss or sl) of the association between adverse environment and negative developmental outcomes. Examples of such studies were the associations between bullying victimization experiences and emotional problems, ${ }^{13}$ between family risk and depression, ${ }^{14}$ between prenatal maternal anxiety and infant negative emotionality ${ }^{15}$ and between early institutional deprivation and emotional problems in adolescence. ${ }^{16}$ Thirty-six effect sizes $(N=3498)$ - enabling a focus on the 'bright side'-pertained 
Table 2 Combined effect sizes for the associations between positive or negative environmental factors and child outcomes for the various 5 HTTLPR genotypes in the total set of studies $(k=77)$

\begin{tabular}{|c|c|c|c|c|c|c|c|c|c|c|c|c|}
\hline \multirow[t]{2}{*}{ Genotype } & \multicolumn{5}{|c|}{ Positive environment } & \multicolumn{5}{|c|}{ Negative environment } & \multicolumn{2}{|c|}{ Contrast } \\
\hline & $\mathbf{K}$ & $\mathbf{N}$ & $\mathbf{r}$ & $95 \% \mathrm{Cl}$ & $\mathbf{Q}$ & $\mathbf{k}$ & $\mathbf{N}$ & $\mathbf{r}$ & $95 \% \mathrm{Cl}$ & $\mathbf{Q}$ & $\mathbf{Q}$ & $\mathbf{P}$ \\
\hline $\begin{array}{l}\text { (1) } \mathrm{ss} \\
\text { (2) } \mathrm{ss} / \mathrm{sl} \\
\text { (3) } \mathrm{sl} \\
\text { (4) } \mathrm{sl} / \mathrm{Il} \\
\text { (5) } \mathrm{II} \\
\text { (6) all ss/sl }(1+2+3)\end{array}$ & $\begin{array}{r}7 \\
9 \\
4 \\
3 \\
13 \\
20\end{array}$ & $\begin{array}{r}312 \\
1004 \\
568 \\
415 \\
1199 \\
1884\end{array}$ & $\begin{array}{l}0.17^{\star \star} \\
0.27^{\star \star} \\
0.17^{\star} \\
0.06^{\star} \\
0.11^{\star \star} \\
0.21^{\star \star}\end{array}$ & $\begin{array}{l}0.07 \sim 0.25 \\
0.09 \sim 0.43 \\
0.01 \sim 0.33 \\
0.01 \sim 0.11 \\
0.04 \sim 0.19 \\
0.12 \sim 0.30\end{array}$ & $\begin{array}{c}5.12 \\
110.81^{\star \star} \\
9.36^{\star} \\
0.33 \\
24.80^{\star} \\
135.48^{\star *}\end{array}$ & $\begin{array}{r}10 \\
6 \\
9 \\
1 \\
15 \\
25\end{array}$ & $\begin{array}{r}715 \\
1312 \\
1772 \\
170 \\
1894 \\
3739\end{array}$ & $\begin{array}{l}0.31^{\star \star} \\
0.09 \\
0.20^{\star \star} \\
0.00 \\
0.06 \\
0.22^{\star \star}\end{array}$ & $\begin{aligned} 0.24 & \sim 0.37 \\
-0.13 & \sim 0.30 \\
0.08 & \sim 0.31 \\
-0.15 & \sim 0.15 \\
-0.01 & \sim 0.12 \\
0.14 & \sim 0.31\end{aligned}$ & $\begin{array}{l}6.51 \\
20.16^{\star \star} \\
30.71^{\star \star} \\
\quad n . a . \\
12.34 \\
79.86^{\star \star}\end{array}$ & $\begin{array}{l}6.17 \\
1.59 \\
0.07 \\
\text { n.a. } \\
1.30 \\
0.03\end{array}$ & $\begin{array}{l}.01 \\
.21 \\
.80 \\
\text { n.a. } \\
.26 \\
.86\end{array}$ \\
\hline
\end{tabular}

${ }^{\star} P<0.05,{ }^{\star *} P<0.01$. Note: direction of effect sizes was labeled according to the a priori hypotheses of this meta-analysis.

to the relation between supportive contexts (for example, warm-responsive parenting) and positive developmental outcomes. These studies concerned, for example, the effect of mothers' positive emotions expressed about their children with attention deficit/hyperactivity disorder, ${ }^{17}$ the effects of a family-centered prevention program on adolescents' risky behaviors, ${ }^{18}$ the decrease in anxiety in response to cognitive behavior therapy ${ }^{19}$ or the association of high-quality family functioning (as opposed to neutral or low-quality family functioning) with adolescent alcohol consumption. ${ }^{20}$ As the 5HTTLPR genotype consists of three variants (ss, sl, and II), studies sometimes reported on the three variants separately, but in other cases the sl group was combined with either the ss or the II group. Thus, each of five possible combinations can be found in the literature (see Table 2). The associations of interest were those between (positive or negative) environment and child outcome within each of the five genotype groups, consisting of carriers of the short (putative 'risk') allele (ss and sl genotypes) and carriers of two long alleles (II). All effect sizes were computed through consensus of two coders (MHvlJ, MBK).

Moderators. We included five moderators to test whether effect sizes varied significantly across moderator categories. First, age was coded in two categories: below 10 years (parallel to a previous meta-analysis on $\mathrm{G} \times \mathrm{E}$ with dopaminerelated genes ${ }^{12}$ ), and 10 years and older. Second, as 5HTTLPR ss genotype has been found associated with higher CSF 5-HIAA levels in African Americans, but with lower levels in Caucasians, ${ }^{21}$ we categorized the studies into those involving $>80 \%$ Caucasian participants, and studies involving more than $20 \%$ other ethnicities. Third, a singlenucleotide polymorphism (SNP) in the $L$ allele (rs25531) has recently led to differentiation between the high functioning $L_{a}$ variant versus the $L_{g}$ variant that is more functionally equivalent to the $S$ allele (the triallelic approach ${ }^{22}$ ). We differentiated between the studies using the traditional biallelic approach and studies applying the triallelic method. Fourth, the method of assessing the environment was categorized into studies using observations, questionnaires or interviews completed by persons other than the children (for example, parents), or self-report questionnaires or interviews. Fifth, the same categorization (observation, other-report, self-report) was used for the assessment of the developmental outcomes. Intercoder reliability was adequate (mean 94\%, range 80-100\%). Disagreements were discussed to reach consensus.
Data synthesis. The Comprehensive Meta-Analysis (CMA) program was used to transform the results of the individual studies (for example, means and s.d. in both genotype groups) into the common metric of correlations and to combine effect sizes. ${ }^{23}$ Heterogeneity across studies was assessed using the $Q$-statistic. As most of our data sets were heterogeneous in their effect sizes, and as random effects models are somewhat more conservative than fixed effects parameters in such cases, combined effect sizes and confidence intervals ( $\mathrm{Cls}$ ) from random effects models are presented. We also tested the influence of the five moderators on the variation of effect sizes between studies with the $Q_{\text {contrast }}$ statistic in a random effects model. ${ }^{23}$ The $Q_{\text {contrast }}$ statistic is based on the logic of analysis of variance, with the total variance $Q_{\text {total }}$ partitioned into $Q_{\text {between }}$ and $Q_{\text {within. }} Q_{\text {total }}$ is the variance with any grouping factors ignored, and $Q_{\text {within }}$ for each group refers to the variances in the specific subsets of studies. $Q_{\text {between }}=Q_{\text {total }}-Q_{\text {within }}$, and is tested for significance using the $\chi^{2}$ distribution. ${ }^{23}$ A significant $Q_{\text {contrast }}$ value indicates that the difference in effect size between subsets of studies is significant.

\section{Results}

In Table 2, the combined effect sizes for each of the five genotype groups are presented. The effect sizes represent the associations between variations in the environmental and developmental outcomes. Here we discuss the contrast between the carriers of one or two $s$ alleles (the combination of the groups with ss, ss/sl or sl carriers) and the carriers of two I alleles (II carriers), separately for the effect of adverse and supportive environments.

Adverse environments. The combined effect size for developmental problems in the presence of negative environmental influences (that is, 'the dark side') amounted to $r=0.22(P<0.01,95 \% \mathrm{Cl} 0.14,0.31)$ for ss/sl carriers, in a heterogeneous set of effect sizes $(Q=79.86, P<0.01)$. The combined effect size for the II carriers was $r=0.06$ (NS, 95\% $\mathrm{Cl}-0.01,0.12)$ in a homogeneous set $(Q=12.34, \mathrm{NS})$. Using a random effects test, the difference was significant $\left(Q_{\text {contrast }}=8.35, P<0.01\right)$, supporting the diathesis-stress idea that $\mathrm{ss} / \mathrm{sl}$ carriers are more vulnerable to environmental adversity than II carriers. 
Positive environments. Turning to the 'bright side'-the association between positive environments and better child outcomes-we found a combined effect size of $r=0.21$. $(P<0.01,95 \% \mathrm{Cl} 0.12,0.30)$ for $\mathrm{ss} / \mathrm{sl}$ carriers in a heterogeneous set of effect sizes $(Q=135.48, P<0.01)$. The combined effect size for II carriers was $r=0.11(P=0.04,95 \%$ Cl $0.04,0.19)$ in a marginally heterogeneous set $(Q=24.80$, $P<0.05$ ). Although this differential pattern of associations was consistent with differential susceptibility, the difference in effect sizes across the two genetic groups was nonsignificant $\left(Q_{\text {contrast }}=0.54, \mathrm{NS}\right)$. Thus, although children with $s$ alleles were more negatively affected by adverse contexts than II carriers with regard to negative outcomes (see above), they did not benefit significantly more from positive environments than children homozygous for the I allele. It should be noted that only in the case of the carriers of the short allele in negative environments did the funnel plot reveal potential publication bias, which, when corrected with the Duval and Tweedie trim and fill method, ${ }^{23}$ changed only marginally the point estimate of the combined effect size.

Moderators. Moderator analyses focused on age, use of triallelic genotyping, the type of assessment of the environment and the outcome were not significant, and thus did not change our main findings of ss/sl carriers being significantly more vulnerable to negative environments, but not profiting significantly more from positive environments compared with II carriers, all $p s>0.14$. However, ethnicity proved to be a significant moderator of the association between positive environmental influences and positive developmental outcome for carriers of the $\|$ alleles $\left(Q_{\text {contrast }}=6.49\right.$, $P=0.01)$.

Caucasian samples. As ethnicity was a significant moderator, we recomputed the combined effect sizes for the ss/ll carriers versus the II carriers using only studies with (mostly) Caucasian participants.

Adverse environments. In the Caucasian samples, the combined effect size for developmental problems in the presence of adverse environmental influences amounted to $r=0.18(P<0.01,95 \% \mathrm{Cl} 0.11,0.25)$ for ss/sl carriers, in a heterogeneous set of effect sizes $(Q=63.06, P<0.01)$. The combined effect size for the II carriers was $r=0.04$ (NS, 95\% $\mathrm{Cl}-0.07,0.14)$ in a homogeneous set $(Q=9.98, \mathrm{NS})$. The difference between the ss/sl versus II carriers was significant $\left(Q_{\text {contrast }}=4.58, P=0.03\right)$. In adverse contexts and consistent with diathesis-stress thinking, ss/sl carriers were more at risk for negative developmental outcomes than II carriers.

Positive environments. The association between positive environments and better child adaptation amounted to a combined effect size of $r=0.17$. $(P<0.01,95 \% \mathrm{Cl} 0.10,0.24)$ for ss/sl carriers, in a heterogeneous set of studies ( $Q=29.26, P<0.01)$, whereas the combined effect size for II carriers was nonsignificant, $r=0.05(95 \% \mathrm{Cl}-0.05$, $0.14)$, in a homogeneous set of studies $(Q=5.73, N S)$. The difference between the ss/sl versus II carriers was significant $\left(Q_{\text {contrast }}=3.92, P=0.048\right)$. Children with ss/sl genotypes

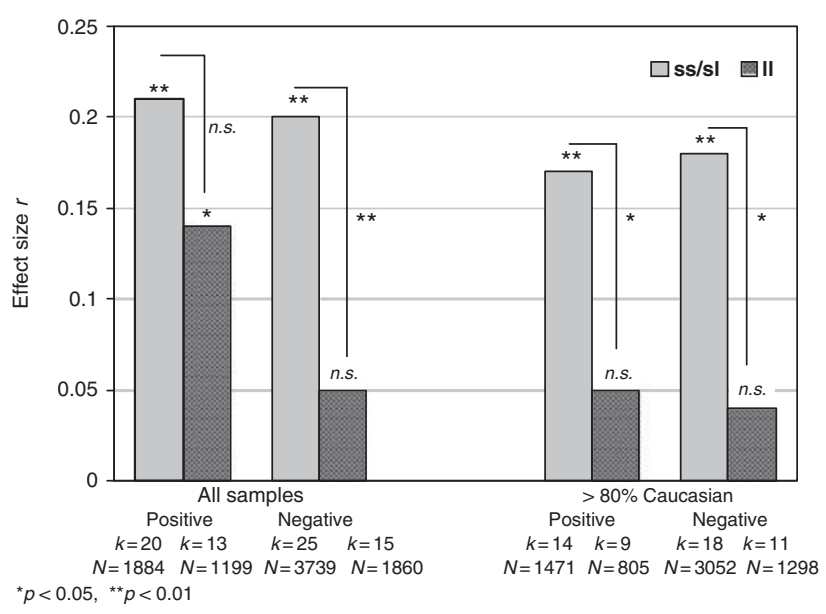

Figure 1 Combined effect sizes for the associations between positive and negative environmental factors and child outcomes for the 5HTTLPR $s \mathrm{~s} / \mathrm{sl}$ and II genotypes, in the total set of studies (except sl/II, $k=73$ ) and in studies with $>80 \%$ Caucasian participants $(k=52)$. ${ }^{*} P<0.05,{ }^{*} P<0.01$. Note: In the total set of studies, the combined effect sizes for positive environmental factors are significant for both $s s / s l$ and II carriers. The difference between the combined effect sizes for $\mathrm{ss} / \mathrm{sl}$ and $/ /$ is not significant, $Q_{\text {contrast }}=0.54, P=0.46$. The combined effect size for negative environmental factors is significant for $s \mathrm{~s} / \mathrm{s} /$ carriers, but not for I/ carriers. The combined effect sizes for $s s / s$ and $/ /$ differ significantly, $Q_{\text {contrast }}=8.35, P<0.01$. In the set of studies with mostly Caucasian participants, the combined effect sizes for positive and negative environmental factors are significant for the $s \mathrm{~s} / \mathrm{s} /$ carriers, but not for the I/ carriers. The differences between the combined effect sizes for $\mathrm{s} / \mathrm{s} /$ and I/carriers are significant, $Q_{\text {contrast }}=3.92, P=0.048$ for positive environmental factors and $Q_{\text {contrast }}=4.58, P=0.03$ for negative environmental factors.

gained more from positive environments than children homozygous for the I allele, consistent with differential susceptibility. In summary, then, for children with the II genotype, the associations between positive or negative environment and positive or negative developmental outcome were absent. Thus, in samples with $>80 \%$ Caucasian children, ss/sl carriers were more open to environmental influences than II carriers, for better and for worse, consistent with differential susceptibility. Figure 1 illustrates these findings.

\section{Discussion}

In this series of meta-analyses on 77 effect sizes with 9361 children and adolescents who were the subject of 30 pertinent research reports, we found some support for 5HTTLPR as a genetic marker of differential susceptibility or plasticity rather than vulnerability. Carriers of the s alleles (ss/sl) were not only at risk for developing poorly in an adverse environment, consistent with the diathesis-stress model, but they were also significantly more influenced by supportive environments enabling them to develop in a more positive way than II carriers, consistent with differential susceptibility. Indeed, the latter finding is incompatible with the diathesis-stress framework that has informed, implicitly or explicitly, most $\mathrm{G} \times \mathrm{E}$ research to date. ${ }^{2,3}$ Ethnicity was an important moderator in the total set of 5 HTTLPR $\mathrm{G} \times \mathrm{E}$ studies involving child and adolescent samples. Some evidence for differential susceptibility only emerged in studies with (mostly) Caucasian participants. Caucasian participants with the II genotype were less influenced by environmental factors, whether supportive 
or adverse. The mixed studies with a higher percentage of non-Caucasians may have resulted in population stratification or admixture, which may seriously elevate the type I error rate for detecting genes underlying complex traits, and in the end may lead to non-replicable $\mathrm{G} \times \mathrm{E}$ findings.

The support for the differential susceptibility theory chronicled here in the case of the 5HTTLPR genotype must be regarded as tentative owing to the fact that the set of studies available for inclusion in our meta-analysis was rather small. Most importantly, the $\mathrm{G} \times \mathrm{E}$ studies conducted thus far are quite heterogeneous in terms of environments and outcomes studied. The heterogeneity creates relatively large confidence intervals $(\mathrm{Cl})$ around the point estimates of combined effect sizes, which may make the meta-analytic results somewhat unstable. More studies using similar assessments of environments and outcomes will afford stronger tests of the hypothesis that 5HTTLPR is a marker of differential susceptibility, the conclusion drawn here. Furthermore, including assessments of positive environments does not exclude the possibility that the resulting associations with (positive) developmental outcomes are driven by the lower scores on these assessments, thereby representing, perhaps, the higher end of negative environmental input. Evidence that carriers of short versus long allele of 5HTTLPR prove more susceptible to experimental interventions promoting development by enhancing the quality of the environment would counter this alternative interpretation.

Most $G \times E$ studies included in the current meta-analyses are correlational in design, making causal inferences impossible. Correlational $\mathrm{G} \times \mathrm{E}$ studies are limited in their control of gene-environment correlations that might wrongly be interpreted as $\mathrm{G} \times \mathrm{E}$ interactions. Only experimental manipulations of the environment in randomized control trials afford valid conclusions about $\mathrm{G} \times \mathrm{E}$ interactions independent of gene-environment correlations. ${ }^{24}$ Protocolled interventions also create standardized and observable changes in the environment; in so doing, they reduce the error component in the measurement of the environment. This should increase the chance of replicable $\mathrm{G} \times \mathrm{E}$ findings, ${ }^{24}$ as these have been found to be greatly dependent on the quality of the assessment of the environment. ${ }^{7}$ Furthermore, in most developmental studies, 5HTTPLPR was the only candidate genetic marker of differential susceptibility. However, differential susceptibility will not depend on one genotype only; other genotypes have already been successfully examined as markers of differential susceptibility (perhaps most compelling dopamine-system related genes ${ }^{12}$ ). In future studies, genetic pathways related to the serotonin system might be included in the $\mathrm{G} \times \mathrm{E}$ equation, and the combined effects of serotonergic and dopaminergic pathways may be examined. This requires studies with large samples that only consortia of researchers are able to conduct.

The diathesis-stress model still dominates the field of developmental psychopathology and psychiatric genetics more generally, stressing the negative developmental impact of a vulnerable genetic make-up interacting with adverse environments. The current meta-analyses show, however, just like our earlier one on dopamine-related genes and child development, ${ }^{12}$ that genetic vulnerability might also imply greater susceptibility to the influences of positive (changes in) environments. At least in the case of children under the age of 18 years, the exclusive 'dark-side' view of some genotypes (for example, 5HTT s allele, DRD4-7 repeat allele) as risk factors for psychopathology appears on the basis of this and our previous meta-analysis undeserved and incompatible with emerging empirical evidence. Similar to the diathesis-stress model, differential susceptibility theory acknowledges the negative effects of cumulative genetic and environmental risks, but at the same time draws attention to the bright side of development. The so-called vulnerability genes might make individuals not only vulnerable to negative environmental influences but also more open to the positive developmental effects of positive changes in the environment.

\section{Conflict of interest}

The authors declare no conflict of interest.

Acknowledgements. MJBK and MHvIJ were supported by awards from the Netherlands Organization for Scientific Research (MJBK: VICI grant no. 453-09003; MHvlJ: SPINOZA prize).

Disclaimer. MJBK and MHvIJ had full access to all of the data in the study and take responsibility for the integrity of the data and the accuracy of the data analysis.

1. Bakermans-Kranenburg MJ, van IJzendoorn MH. Genetic vulnerability or differential susceptibility in child development: the case of attachment. J Child Psychol Psychiatry 2007; 48: 1160-1173

2. Belsky J, Bakermans-Kranenburg MJ, van IJzendoorn MH. For better and for worse differential susceptibility to environmental influences. Psychol Sci 2007; 16: 300-305

3. Belsky J, Jonassaint C, Pluess M, Stanton M, Brummett B, Williams R. Vulnerability genes or plasticity genes? Mol Psychiatry 2009; 14: 746-754

4. Homberg JR, Lesch KP. Looking on the bright side of serotonin transporter gene variation. Biol Psychiatry 2011; 69: 513-519.

5. Caspi A, Sugden K, Moffitt TE, Taylor A, Craig IW, Harrington $\mathrm{H}$ et al. Influence of life stress on depression: moderation by a polymorphism in the 5-HTT gene. Science 2003; 301: 386-389.

6. Munafò MR, Durrant C, Lewis G, Flint J. Gene X environment interactions at the serotonin transporter locus. Biol Psychiatry 2009; 65: 211-219.

7. Uher R, McGuffin $P$. The moderation by the serotonin transporter gene of environmental adversity in the etiology of depression: 2009 update. Mol Psychiatry 2010; 15: 18-22.

8. Belsky J. Variation in susceptibility to environmental influence an evolutionary argument. Psychol Inquiry 1997; 8: 182-186.

9. Belsky J, Pluess M. The nature (and nurture?) of plasticity in early human development. Perspect Psychol Sci 2009; 4: 345-351.

10. Ellis BJ, Boyce WT, Belsky J, Bakermans-Kranenburg MJ, van IJzendoorn MH. Differential susceptibility to the environment: an evolutionary-neurodevelopmental theory. Dev Psychopathol 2011; 23: 7-28.

11. Bakermans-Kranenburg MJ, van IJzendoorn MH. Gene-environment interaction of the Dopamine D4 Receptor (DRD4) and observed maternal insensitivity predicting externalizing behavior in preschoolers. Dev Psychobiol 2006; 48: 406-409.

12. Bakermans-Kranenburg MJ, van IJzendoorn MH. Differential susceptibility to rearing environment depending on dopamine-related genes: new evidence and a meta-analysis. Dev Psychopathol 2011; 23: 39-52.

13. Sugden K, Arseneault L, Harrington H, Moffitt TE, Williams B, Caspi A. Serotonin transporter gene moderates the development of emotional problems among children following bullying victimization. J Am Acad Child Adolesc Psychiatry 2010; 49: 830-840.

14. Eley TC, Sugden K, Corsico A, Gregory AM, Sham P, McGuffin P. et al. Gene-environment interaction analysis of serotonin system markers with adolescent depression. $\mathrm{Mol}$ Psychiatry 2004; 9: 908-915.

15. Pluess M, Velders FP, Belsky J, van IJzendoorn MH, Bakermans-Kranenburg MJ, Jaddoe VW et al. Serotonin transporter polymorphism moderates effects of prenatal maternal anxiety on infant negative emotionality. Biol Psychiatry 2011; 69: 520-525.

16. Kumsta R, Stevens S, Brookes K, Schlotz W, Castle J, Beckett C et al. 5HTT genotype moderates the influence of early institutional deprivation on emotional problems in adolescence: evidence from the English and Romanian Adoptee (ERA) study. J Child Psychol Psychiatry 2010; 51: 755-762. 
17. Sonuga-Barke EJS, Oades RD, Psychogiou L, Chen W, Franke B, Buitelaar J et al Dopamine and serotonin transporter genotypes moderate sensitivity to maternal expressed emotion: the case of conduct and emotional problems in attention deficit/hyperactivity disorder. J Child Psychol Psychiatry 2009; 50: 1052-1063.

18. Brody GH, Chen Y-F, Beach SRH, Philibert R, Kogan SM. Participation in a familycentered prevention program decreases genetic risk for adolescents' risky behaviors. Pediatrics 2009; 124: 911-917.

19. Eley TC, Hudson JL, Creswell C, Tropeano M, Lester KJ, Cooper P et al. Therapygenetics: the 5HTTLPR and response to psychological therapy. Mol Psychiatry 2012; 17: 236-237.

20. Nilsson KW, Sjöberg RL, Damberg M, Alm PO, Ohrvik J, Leppert J et al. Role of the serotonin transporter gene and family function in adolescent alcohol consumption. Alcohol Clin Exp Res 2005; 29: 564-570.

21. Williams RB, Marchuk D, Gadde KM, Barefoot JC, Grichnik K, Helms MJ et al. Serotoninrelated gene polymorphisms and central nervous system serotonin function Neuropsychopharmacology 2003; 28: 533-541.
22. Hu X-Z, Lipsky RH, Zhu G, Akhtar LA, Taubman J, Greenberg BD et al. Serotonin transporter promoter gain-of-function genotypes are linked to obsessive-compulsive disorder. Am J Human Genet 2006; 78: 815-826.

23. Borenstein M, Hedges LV, Higgins JPT. Introduction to meta-analysis. Chicester, NH: Wiley, 2009.

24. van IJzendoorn MH, Bakermans-Kranenburg MJ, Belsky J, Beach S, Brody G, Dodge KA et al. Gene-by-environment experiments: a new approach to finding the missing heritability. Nat Rev Genet 2011; 12: 881-882.

(c) Translational Psychiatry is an open-access journal SOMERIHHISRESERVED published by Nature Publishing Group. This work is licensed under the Creative Commons Attribution-Noncommercial-No Derivative Works 3.0 Unported License. To view a copy of this license, visit http://creativecommons.org/licenses/by-nc-nd/3.0/ 